\title{
Optics education for now and future from an entropy perspective
}

\section{Shoang Donn}

Shoang C. Donn, "Optics education for now and future from an entropy perspective," Proc. SPIE 2525, 1995 International Conference on Education in Optics, (13 October 1995); doi: 10.1117/12.224075

SPIE Event: SPIE's 1995 International Symposium on Optical Science, Engineering, and Instrumentation, 1995, San Diego, CA, United States 


\title{
Optics Education for Now and Future \\ from an Entropy Perspective
}

\author{
Shoang C. Donn \\ Department of Physics \\ Chung Yuan University \\ Chung-Li, Taiwan, R.O.C. \\ email: donn@phys730.cycu.edu.tw
}

\begin{abstract}
During the $20^{\text {th }}$ century, the electronics industry has evolved from radio, television, and audio systems to computer systems. The trace of evolution is clearly from linear systems to nonlinear systems. This is by no means accidental, and is due to the fact that nonlinear systems are more versatile than linear systems. As of now, the successful commercial optical instruments are all linear systems. From the entropy point of view, as the knowledge of human beings further expands, we need more powerful computing capabilities such as memory association and self organization. Based on the fundamental differences between the electron and the photon, this paper presents the argument that optical computing will provide memory association and self organization ability (which compliments the current electronic logical computing). Thus, one can infer that the main future thrust of the optical industry will be in nonlinear optical systems and hybrid combinations with nonlinear electronic systems in order to provide both self organization and logical computing (to emulate, more or less, the computing power of the human brain). Currently, the state of the art has already demonstrated successful combinations of linear optical systems with nonlinear electronic systems (computers). These kinds of hybrid systems are called electro-photonic systems in the following curriculum.
\end{abstract}

Based on the above vision for the future and taking into account the need for immediate employment, the following curriculum is designed to provide a core training to the students who are interested in choosing optics or electro-photonics as their career. This curriculum is being offered in the upper years at the Physics Department of Chung Yuan University.

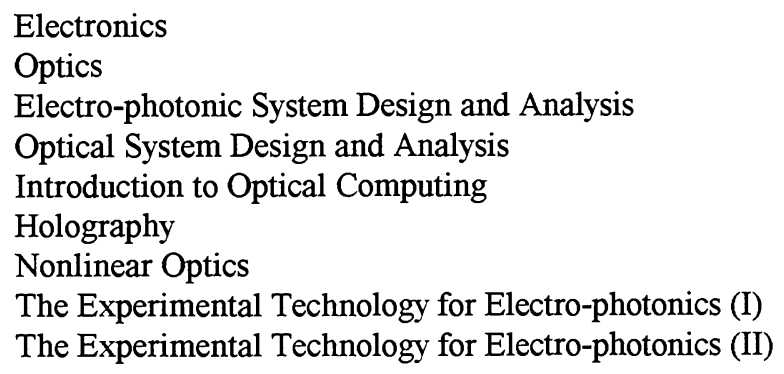

The main thrust among all these courses is the system modeling and analysis. In this paper, the design of this curriculum will be elaborated and the topics for each course will be listed in detail.

Keywords: Electro-optics training, electro-photonics, information entropy, electronic system, photonic system, linear system, non-linear system, neural network, computation, information processing 


\section{Introduction}

Optics has a profound application in many areas, such as medicine, communication, information processing, mechanical and structural engineering, and so on. Thus, optics in conjunction with some interdisciplinary training offers ample career opportunities. To design a curriculum in such a wide-open field in order to prepare students for a career in these areas, one must set forth a core curriculum for basic training and provide additional interdisciplinary training for each chosen field. To this end, one must then consider the trend of technology and the needs of society. The needs of society will dictate the training that students must undertake in order for them to obtain employment. The trend of technology will help cultivate the research ability and motivation of the students. This paper will examine the needs of society and the trend of technology, then justify the curriculum design and provide a detailed description of each course. The needs of society can be found in connection with the entropy of human knowledge and the ability to process information. In fact, this observation has dictated not only the needs of society but also the trend of technology. Thus, we will start with examining the historical evolution of technology and provide a vision for future trends based on this entropy perspective of human knowledge.

\section{Historical Review}

Today the computer has become an integral part of daily life. Not too long ago, it was still a special tool for research and industry and was only used by a handful of professionals. It has become so popular today because it is able to process vast amounts of information in a timely manner and in arbitrary fashion. Why is it so versatile? The answer is "it is a kind of non-linear system." Recall the fact that the output of a linear dispersive system is the point spread function convolved with its input. The output of a non-linear system, however, can be arbitrarily coupled to its input. For a computer system, this non-linearity is derived from its programmability. More specifically, the relation between the output and the input can be changed upon demand through programming and can be executed repeatedly. Thus, this repeatable non-linearity provides the versatility necessary to manage the volume and the complexity resulting from the entropy increase of human knowledge. As one can easily observe from Table 1 the electronics industry throughout the $20^{\text {th }}$ century has been evolving from linear systems to non-linear systems in an effort to cope with this increasing entropy. 
Table 1. Electronic Technology Evolution during the $20^{\text {th }}$ Century

\begin{tabular}{|c|c|c|c|}
\hline YEAR & RADIO & TELEVISION & COMPUTER \\
\hline 1895-1907 & \multicolumn{3}{|c|}{ VACUUM TUBE } \\
\hline 1920 & $\begin{array}{l}\text { COMMERCIAL } \\
\text { BROADCASTING }\end{array}$ & & \\
\hline 1929 & \multicolumn{3}{|c|}{ SEMICONDUCTOR } \\
\hline 1937 & & $\begin{array}{l}\text { COMMERCIAL } \\
\text { BROADCASTING }\end{array}$ & \\
\hline $1940-1949$ & & & PROGRAMMING \\
\hline 1946 & & & ENIAC (vacuum tube) \\
\hline 1956 & & & SEMICONDUCTOR \\
\hline $1960-1970$ & & & IC \\
\hline 1980 & & & VLSI \\
\hline 1990 & & & AI \\
\hline
\end{tabular}

On the optics side, the telescope and the microscope have been in use for centuries but the optics industry lacks the progress which has been made in its electronics counterpart. One may rush to the conclusion that if we convert all electronic devices and circuits to optical equivalents, we may advance information processing technology and hence replay the electronics boom in the optics industry. In fact, this is exactly what the goal is in the continuing development of the "optical computer", which employs the traditional von Neuman architecture. But this approach does not seem to promise the computational capability required to solve random (non-algorithm definable) problems. Thus, one has to carefully examine the fundamental differences between the photon and the electron and then take advantage of their inherent natures in applying them to information processing. This leads to the discussion of the following section.

\section{Technology Trend and the Needs of Hybridizing}

Table 2 lists the fundamental differences between the photon and the electron, and their advantages and disadvantages. Photons are bosons and there are no mutual interactions among them, thus, photons can propagate in parallel and cross each other freely in three-dimensional space at the speed of light $c$. Electrons are fermions and are subject to a mutual repulsion force, thus, they can only propagate along a twodimensional surface in sequence. This sequential propagation property of the electron restricts the fundamental electronic system to sequential processing (though certain limited forms of parallelization can be achieved at a relatively high cost, optical parallel processing comes by more naturally and is more economical). This, in turn, prescribes the problem domain of electronic information processing systems. Since electronic computers require "programming" to process information, the type of problems that an electronic computer can solve most efficiently are structured (algorithm definable) problems. On the other hand, one can process information in parallel in a photonic system because of the parallel propagation capability of the photon. Furthermore, such parallel propagation capability is bi-directional(c.f. electronic sequential propagation is uni-directional) which is important for the memory association and the self-organization 
capabilities mentioned below. As such, photonic systems have the potential to solve more complicated, greater entropy problems, especially problems not easily algorithm definable, for instance, pattern recognition. It has been shown that optical implementations of neural networks coupled with a non-linear threshold phaseconjugator are well suited for performing memory associations and hence pattern recognition ${ }^{1}$. Furthermore, the self-organization capability that provides self-learning in such problem solving can be accomplished via instantaneous adjustment of the interconnections in an optical neural network using appropriate crystals to store dynamic holograms $\mathrm{s}^{2,3.4}$. Thus photonic systems have promising capabilities unparalleled by their electronic counterparts. This does not preclude the possibility of realizing an optical computer with a von Neuman architecture, however, such an implementation would not take full advantage of the photon.

Table 2. Fundamental Differences between the Electron and the Photon

\begin{tabular}{|l|l|l|}
\hline & Electron & Photon \\
\hline Particle Type & Fermion & Boson \\
\hline Freedom of Propagation & 2-dimension & 3-dimension \\
\hline Propagation Mode & Sequential & Parallel \\
\hline Problem Type & Structured & Random \\
\hline
\end{tabular}

As society evolves, there is a constant need for better information processing capability. The ever increasing needs of humanity are the driving forces behind the expansion of knowledge and hence, processing capability. This has been proven throughout the evolution of the computer in the past half century. Thus, the trend of technology is moving toward offering the capability of processing problems with larger and larger entropy.

Yet the state of the art has not fully realized the power of photonics. More specifically, we have not perfected the art of making non-linear optical systems. However, we do build very useful linear optical systems and non-linear electronic systems today. The best course, then, is to build hybrids combining both electronic and photonic processing systems. For instance, bio-fluorescence analyzers and fibercommunication networks contain both electronic and photonic sub-systems which harness the current state of technology. This is achievable because the electronic and optical parts are complimentary to each other. This hybridizing (along with another combination) is illustrated in the following diagram. 


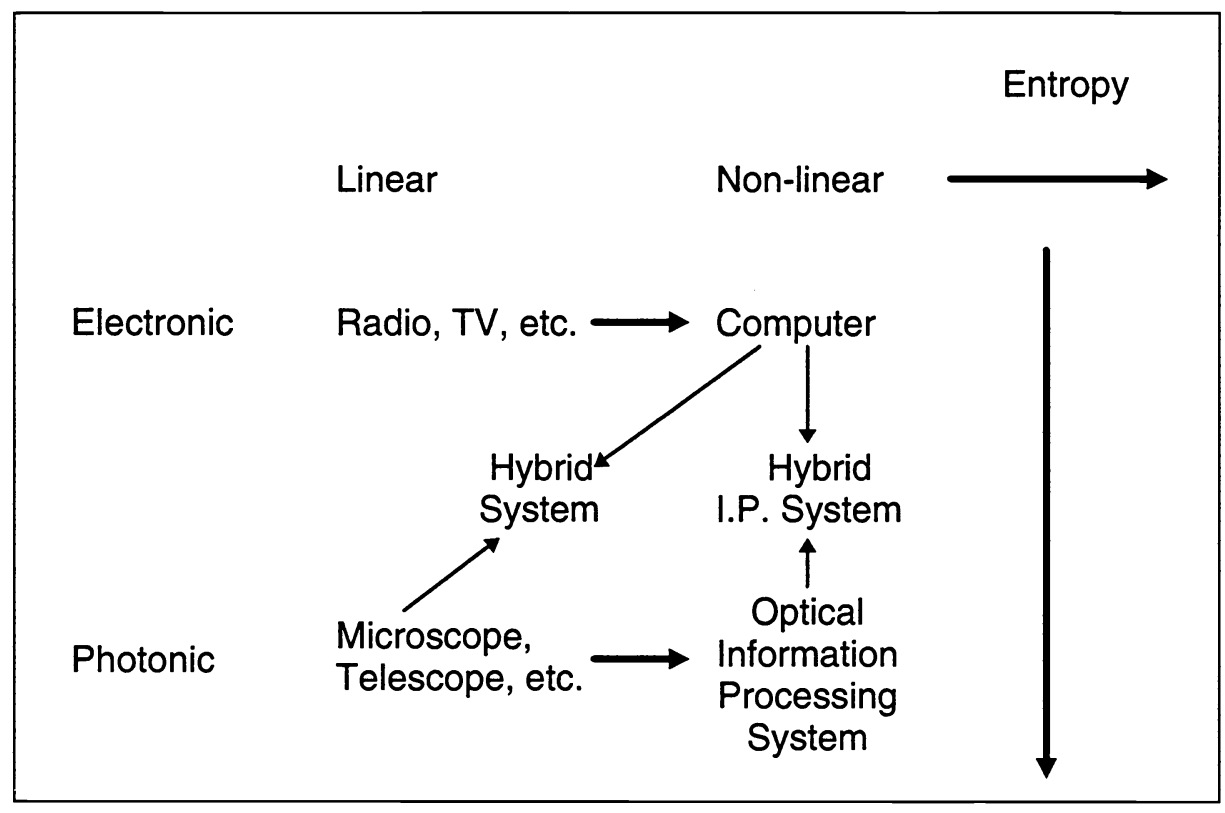

Fig. 1 Technology Evolution and Trends

Here, the entropy increases along both the horizontal and the vertical axes, i.e., nonlinear systems can process problems with greater entropy than linear systems, and likewise, photonic systems can process problems with greater entropy than electronic systems. We use the term "optical information processing system" but not "optical computer" here to indicate that such a system is not necessarily of a von Neuman architecture and in fact may exist in various forms. As future requirements dictate, one may even have to build hybrids combining non-linear photonic systems and non-linear electronic computers. This is labeled the hybrid I.P. system in the lower right of the diagram.

\section{Fundamental Thrust}

The hybridization between electronics and photonics is a wide open discipline that seems has no well-defined domain. Moreover, various professionals from similar overlapping fields employ the terms "optoelectronics", "optronics"5 "photonics", "electro-optics", etc., in describing their own work, depending on their perspective. Among these terms, "electro-optics" is most commonly used to describe the hybridization. But if we confine ourselves to the above entropy perspective, we would like to suggest a new term "electro-photonics", in contrast to electro-optics (which has origins linked to the electro-optical effect). Since the technology is harnessed at the stage of system integration, the basic thrust of this interdisciplinary training program is set on the system perspective. Even if students intend to pursue a career in the component side of the technology, a system perspective can provide them with a wider horizon and perhaps some good reasons and good motivation for pursuing their chosen field. 
Thus, in this curriculum, we concentrate on dealing with the system aspect of this technology. The fundamental theoretical blocks of all signal (information) processing systems: Fourier transform, impulse response, system transfer function, convolution, correlation, dispersion and its implication on impulse response, etc. form the central theme throughout the curriculum. In fact, this methodology is applicable to all systems, whether electronic or photonic. These subjects are thoroughly discussed in the course entitled "Electro-photonic System Design and Analysis". This is one of the core courses of the whole curriculum. Once students master these basic concepts, they can apply them in other courses such as "Optical System Design and Analysis", "Holography", "Nonlinear Optics", and so on.

\section{Curriculum Design}

In order to realize the vision described above, the curriculum is designed so that students first complete a minimum set of fundamental and core courses, then are allowed to branch to an engineering or a research path. The research students are, however, encouraged to complete the engineering courses as well in order to establish a solid foundation. The following diagram illustrates the recommended course flow sequence for students. The solid lines indicate the flow of the sequence and the dashed lines indicate alternate flows of the sequence, while the dotted lines indicate two courses that must be taken concurrently in order to derive maximum benefit from this program. 


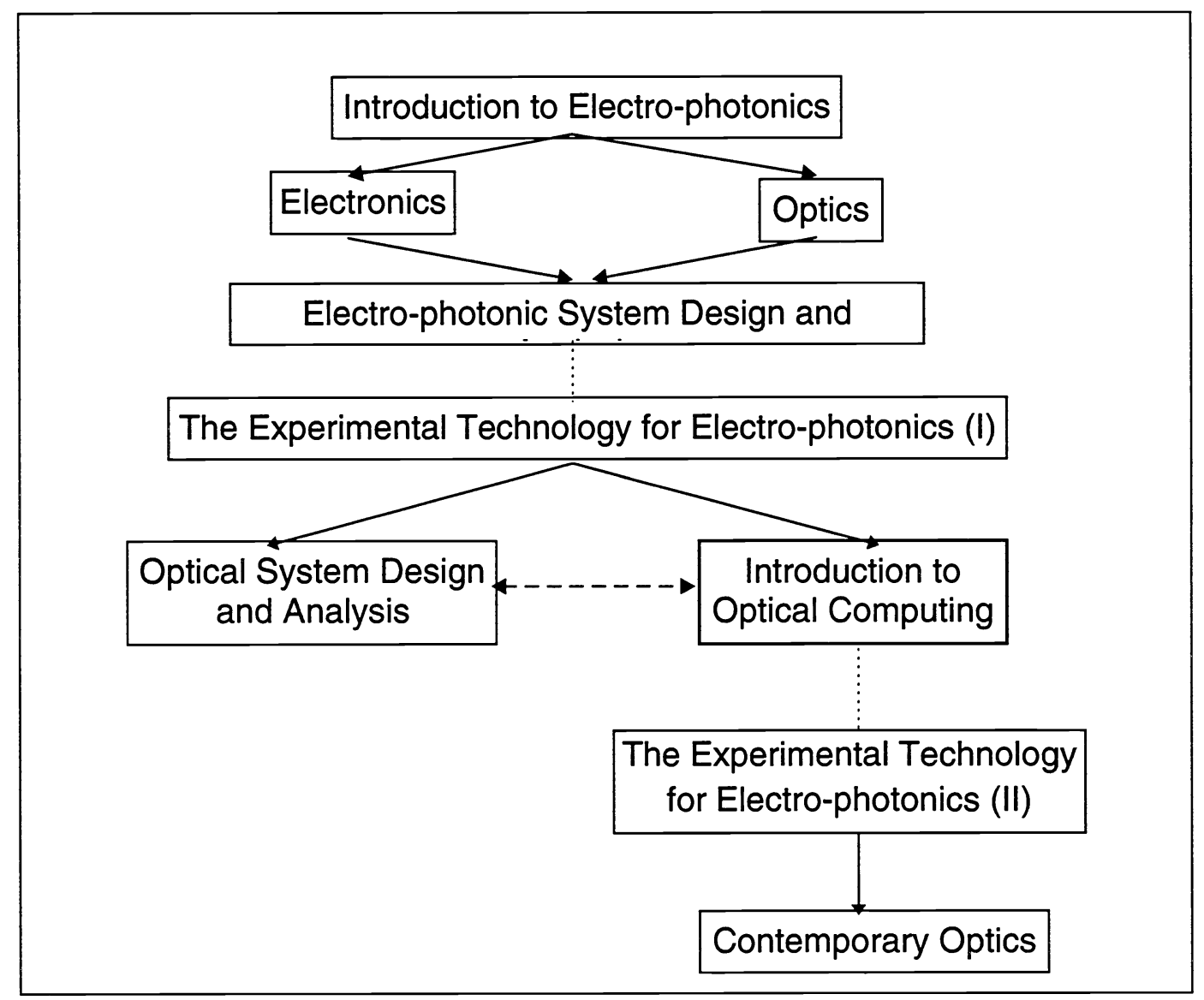

Fig. 2 Recommended Course Sequence Flow Chart

The first course of this curriculum, entitled "Introduction to Electro-photonics", serves as the introduction to the whole program and provides an overview of the entire sequence. If after this exposure, the students choose to pursue this Electro-photonic program then they must start with the two fundamental courses: Electronics, and Optics (they may study these two courses without going through the above introductory course). After they learn the basic concepts of these two subjects. they then have to take the core course "Electro-photonic System Design and Analysis" in order to master those central theme subjects mentioned in the previous section. Electronics and optics are brought together into one unified perspective in this course. In addition, the course also covers sampling and quantization. Learning these materials can be enhanced by concurrently taking the second core course entitled "The Experimental Technology for Electrophotonics(I)". In this course, among a few other basic experiments, students implement signal quantization, computer interfacing, and signal reconstruction in a related series of laboratory sessions. Upon completion of these two core courses, students should be able to design and construct the hybrid system described in Section 3. Afterwards, study can be concentrated on the engineering option or the research option. If the first option is chosen, they can study "Optical System Design and Analysis" in which the central theme of signal processing is applied to optical system analysis in addition to basic topics such as light tracing, thin film design, aberration control and correction. With "Optical 
System Design and Analysis ", this training covers the current state of technology. Students can further broaden their education by taking additional courses from the list of Table 3 (explained in detail later) according to their interests.

Those who are interested in next-generation technology (the research path) will have to include "Introduction to Optical Computing", "The Experimental Technology for Electro-photonics (II) " and "Contemporary Optics" in their studies. In the first course, the central theme of signal processing is applied to various forms of optical signal processing and computing systems. Holography, non-linear optics, and "digital optical computers" are also introduced in this course. "The Experimental Technology for Electro-photonics (II) " offers optical signal processing, fabrication of various types of holograms, experimentation with lasers, and other advanced experiments. At the graduate level, one can take "Holography" and "Nonlinear Optics", both of which are combined into one year-long course entitled "Contemporary Optics".

There are two courses not illustrated in the diagram; one is entitled "Optoelectronics", and the other is entitled "Lasers and their Applications". The first one introduces various optoelectronic components to the student while the second course provides the student with the facts and theory they need to work with lasers, along with example applications.

Table 3 lists these courses along with the year and the semester that they are offered. Each course is classified as "Fundamental", "Core,", "Main", or "Auxiliary" in the first column.. "Electronics" and "Optics" are listed as the fundamental courses, which students must complete before they can enter the program. Both "Main" and "Auxiliary" indicate those courses that are optional, while the "Core" are those courses which must be taken in order to complete the program. The "Main" and "Core" courses are those recommended in the above course sequence flow chart.

Table 3. List of Course Offerings by Year

\begin{tabular}{|l|l|l|}
\hline Main & Introduction to Electro-photonics & Sophomore \\
\hline Fundamental & Electronics & Sophomore \\
\hline Fundamental & Optics & 1st Semester of Junior \\
\hline Core & $\begin{array}{l}\text { Electro-photonic System Design and } \\
\text { Analysis }\end{array}$ & 2nd Semester of Junior \\
\hline Core & $\begin{array}{l}\text { The Experimental Technology for } \\
\text { Electro-photonics (I) }\end{array}$ & 1st Semester of Senior \\
\hline Auxiliary & Optoelectronics & 2nd Semester of Junior \\
\hline Main & Introduction to Optical Computing & 1st Semester of Senior \\
\hline Main & $\begin{array}{l}\text { The Experimental Technology for } \\
\text { Electro-photonics (II) }\end{array}$ & 1st Semester of Senior \\
\hline Main & Optical System Design and Analysis & 2nd Semester of Senior \\
\hline Auxiliary & Laser and its Application & 2nd Semester of Senior \\
\hline Main & Contemporary Optics & 1st Year Graduate School \\
\hline
\end{tabular}


All of the courses listed except the last one are one-semester courses. This curriculum is offered in the graduate school and the upper years of the undergraduate school at the Physics Department of Chung Yuan University. The core courses also serve as the core curriculum of a much broader, campus-wide Interdisciplinary Electro-photonics Program, which is one example of the interdisciplinary training mentioned in Section 1.

\section{Course Descriptions}

Table 4 summarizes the major topics for each of the main and core courses. The tables of contents for selected subjects follow.

Table 4. Major Topics for Main Courses

\begin{tabular}{|l|l|}
\hline Course & Major Topics \\
\hline Electronics & RC Circuits, Op Amp, Digital Electronics \\
\hline Optics & $\begin{array}{l}\text { Waves, EM Waves, The Interaction of Light } \\
\text { and Matter }\end{array}$ \\
\hline $\begin{array}{l}\text { Electro-photonic System Design and } \\
\text { Analysis }\end{array}$ & $\begin{array}{l}\text { Fourier transform, convolution, dispersion, } \\
\text { correlation, system modeling, noise modeling, } \\
\text { sampling and quantization }\end{array}$ \\
\hline Optical System Design and Analysis & $\begin{array}{l}\text { Paraxial Optics, Meridional Optics, 3D Optics } \\
\text { Light Tracing } \\
\text { Aberration, Thin Film Design } \\
\text { Physical Optics }\end{array}$ \\
\hline Introduction to Optical Computing & $\begin{array}{l}\text { Optical Signal Processing, Holography } \\
\text { Numerical Processing } \\
\text { Switching and Devices }\end{array}$ \\
\hline Contemporary Optics & $\begin{array}{l}\text { Holographic System } \\
\text { Nonlinear Susceptibility } \\
\text { Intensity-Dependent Refractive Index } \\
\text { Electro-optical effect } \\
\text { Photorefraction } \\
\text { Chaos }\end{array}$ \\
\hline
\end{tabular}

\section{ELECTRONICS}

1. RC circuitry

2. Operational Amplifiers

3. Practical Aspects of Operational Amplifiers

4. Transmission Lines

5. Filter Design

6. Digital Electronics 
OPTICS

PART I. WAVE PHENOMENON IN OPTICS

1. Wave Motion

2. Electromagnetic Waves

3. Two Beam Interference

4. Multiple Beam Interference

5. Thin Films

6. Interference By Division Of Wave Front

7. Diffraction

PART II. THE INTERACTION OF LIGHT AND MATTER

1. Absorption and Dispersion

2. Crystal Optics

3. Nonlinear Optics

\section{ELECTRO-PHOTONIC SYSTEM DESIGN AND ANALYSIS}

1. Background in Mathematics

2. System Modeling and Noise Modeling

3. Light Detection

4. Sampling and Quantization

5. Property of Light Sources

6. Optical System Modeling

7. Electronic Circuit Modeling

8. System Analysis

\section{OPTICAL SYSTEM DESIGN AND ANALYSIS}

1. Paraxial Matrix Optics

2. Meridional Rays Optics

3. Three-Dimensional Optics

4. Aberration

5. Optical Instruments

6. Physical Optics and Application to System Analysis

7. Thin Film Design

\section{INTRODUCTION TO OPTICAL COMPUTING}

1. Introduction

2. Background In Optics

3. Holography

4. Optical Image and Signal Processing

5. Optical Numerical Processing

6. Digital Optical Computing, Optical Switching, Memory 


\section{Conclusions}

We have reviewed the historical evolution of the information technology field, and examined the implications of the fundamental differences between the photon and the electron. Based on the increasing information entropy driven by human demand and current technological levels, we have thus provided a vision for future technology trends. Accordingly, we have designed a curriculum for training students in the electro-photonic fields with the aim of meeting society's needs for now and for the future. This Electrophotonic program offers flexibility that allows students to tailor the program of study according to their interests.

\section{Acknowledgements}

The author is indebted to the Ministry of Education and the National Science Council of the Republic of China, and the College of Science and the Department of Physics of Chung Yuan University for their continuous funding and support to make this curriculum formulation and implementation possible. The author is also indebted to Dean B. T. Chung, Chairman M. C. Huang, and all of my colleagues for their continuous support.

\section{References}

1. B.H. Soffer, G.J. Dunning, E. Marom, and Y.Owechko, Appl. Opt. 26,1900(1987).

2. Clark C. Guset et al. , Appl. Opt. 26,5055(1987)

3. K. Wagner and D. Psaltis, Appl. Opt. 26,5061(1987)

4. Robert A. Senn et al. , Appl. Opt. 29,1591(1990).

5. Brian J. Thompson,. "Education in Optics - Challenges at Hand", Proc. SPIE, Vol.. 978,2-9(1988). 